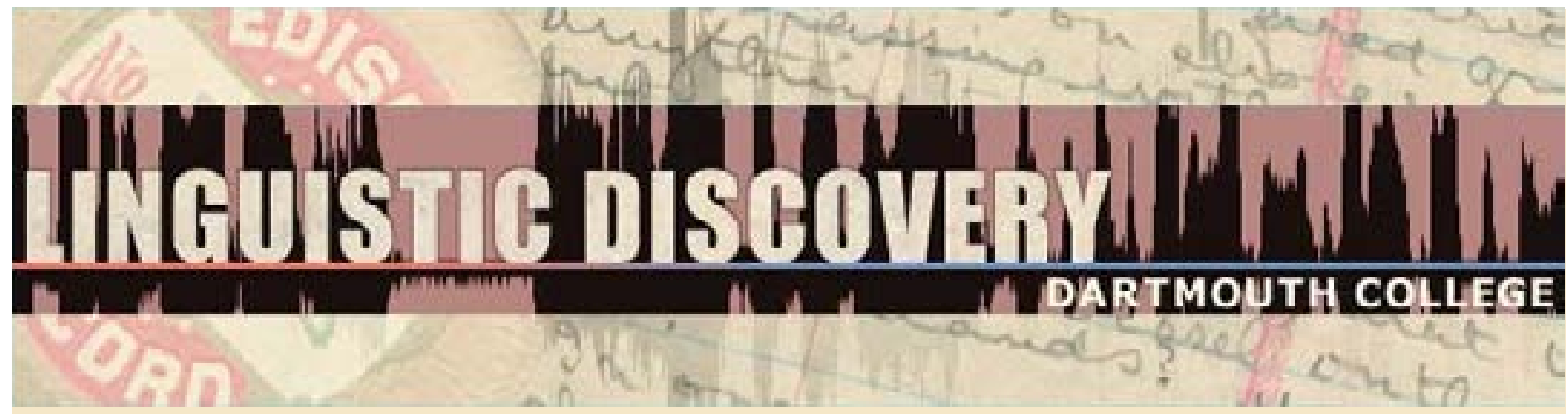

\begin{tabular}{|l|}
\hline Volume 10 \\
Issue 2 \\
2012 \\
\hline
\end{tabular}

\title{
Persian Back Channel Responses in Formal versus Informal Contexts
}

Shahla Sharifi \& Mahnaz Azadmanesh

Ferdowsi University of Mashhad, Iran

doi: 10.1349/PS1.1537-0852.A.401

url: http://journals.dartmouth.edu/cgi-bin/WebObjects/ Journals.woa/1/xmlpage/1/article/401 


\section{Persian Back Channel Responses in Formal versus Informal Contexts \\ Shahla Sharifi \& Mahnaz Azadmanesh \\ Ferdowsi University of Mashhad, Iran}

Utterances like /xob/ (okay), /doroste/ (right), /hmm/, lee/, /âre/ (yeah), occur frequently in Persian conversations, but have thus far escaped from the systematic studies. Good listeners generally produce these short utterances, called "back channel responses", in appropriate times to show their participation in the conversation, but the rules governing back channeling vary from one context to another. The usage of back channel responses is different in various contexts, due to politeness or formality. This paper studies the types and functions of the back channel responses in both formal and informal settings and provides a comparison of the usage of these responses in these two kinds of contexts. The results show /bale/ (yes) and /doroste/ (right) are used with formal or polite verbal form, while /xob/ (okay) is used with the informal style of speech and less polite verbal form. With respect to the function of back channels, signaling the understanding is the main function of back channels in informal contexts. Also, back channels signaling agreement are more frequent in formal contexts, where emotional function is less likely.

\section{Introduction}

Back channel responses (BC) are pervasive phenomena in the human communicative behavior, because participants in a conversation continuously give or elicit back channels as a way of explicitly exchanging information about the state of communication, for instance to show attention, understanding, misunderstanding, acceptance or non-acceptance, in order to make communication more efficient. The study of back channel responses is relevantly new, perhaps with the advent of new technology, in 1950s, the investigation of spoken language and listeners' behavior was possible. One of the first authors who have noticed and described some of the communicative behaviors that nowadays we call them "back channels" was Fries(1952) who analyzed a corpus of telephone conversations in which he recognized as series of "listener response". In 1970, it was Yngve (1970) who first coined the term back channel to describe these tokens. After that, particular attention was paid to the scientific examination of these short utterances.

Researchers have identified linguistics differences with regard to the frequency, type, usage, and placement of back channels across languages. Li (2006) studied Chinese and Canadian speech and reported that listener made more back channel responses, whether the Chinese talked with another Chinese or with a Canadian. The finding of his study was in disagreement with earlier studies. Li (2006) found that Chinese/ Chinese group exhibited the highest frequency of back channel responses, the Canadian/ Canadian group the lowest, with the two inter-cultural groups in between. Inversely, Tao \& Thompson (1991) found that English speakers had a higher frequency of back channel responses than Chinese speakers. Li et al. (2010) extended his previous studies and examined the types of back channel responses as well as their relationship with speaker presentation, listener recall, and participants' perceived enjoyment of the intercultural conversations. The results of this study were consistent with $\mathrm{Li}$ (2006). Moreover, they found a negative correlation between the frequency of back channel responses and enjoyment of the conversation. 
White (1997) also examined the effect of Japanese versus American culture on the production of back channel responses. White (1997) concluded that cultural differences between the United States and Japan, regarding politeness and face concern, are responsible for the differences in the usage and function of back channel responses in American English and Japanese. These differences are not confined to unrelated languages, even in languages with similar cultures and histories, these differences are obvious. Kim (2009) discussed the importance of comparative studies of Korean and Japanese linguistics behavior and examined the usage of back channel responses and pause fillers in these groups. According to Kim (2009), Japanese speaker used back channels more frequently than pause fillers, while the reverse is true for Korean speakers, indicating the fact that the role of the listener is more important in Japanese while the role of the speaker is more emphasized in Korean.

Some scholars addressed the transfer of back channel behaviors in bilingual speakers within the accommodation theory frame work. Accommodation theory offers a sound framework for the study of conversational strategies in interpersonal encounters. From an accommodation theory perspective, it would seem that balanced bilingual speaker tend to converge with other native speakers of their first language when they are engaged in a friendly conversation. Heinz (2003) examined the differences of back channel behavior in interactions between monolingual and bilingual Germans. He found significant differences in the frequency and placement of back channel responses among monolingual German speakers and monolingual American English speakers. The author also reported that native Germans, who have become proficient in American English, use more back channel responses and more often in overlapping positions than monolingual Germans do. These results show a contradiction of accommodation theory, but the findings of Li (2006) study provided a support for accommodation theory, which stated that Chinese and Canadian speakers had a tendency to converge their linguistics codes in conversation. Also, Tao \& Thompson (1991) earlier had reported that native Chinese who were fluent in English had a tendency to switch codes, using English back channel responses.

Instead of comparing back channel behavior in different languages, numerous linguistics researchers have focused on the usage of gender specific back channels. Coates (2003), in summing previous research works states that women are said to be more polite, more cooperative and made use of more back channels in conversation than men. Men, on the other hand, are said to follow strategies of non-cooperative, including interruption and less back channels. Previous studies of gender differences were in disagreement with the claims of Coates (2003). Dixon \& Foster (1998) reported that men use more supporting back channel signals than women do when addressing a female audience in South Africa.

Besides the effects of language, culture and gender, different communicative context or different styles of speech had an influence on the back channel behavior. Accordingly Kok \& Heylen (2010) compared the listeners' behaviors elicited by procedural and narrative tasks. The results of this study showed that long procedural tasks elicited more responses than the short tasks, due to the cognitive load of the interlocutors. Furthermore, Angles et al. (2000) referred to the influence of the level of formality and asserted that Japanese tokens $\underline{h a i}$, ee, and $\underline{u n}$ are used in different context, $\underline{h a i}$, and $\underline{\text { ee }}$ are used in informal context, while $\underline{u n}$ is used with casual speech style.

Therefore, back channel responses are a pervasive feature of conversations and as a listener, we must have the ability to produce back channels timely and appropriately. It has been long assumed that there are some places in the dialog where back channels are welcomed. For example, Ward \& Tesukahara (2000) have truly claimed that speakers' cues accounted for about 
half of the occurrences of back channels, suggesting that back channel responses are not elicited whenever the listener liked them, but are encouraged by the current speaker. Ward (1996) believed that a low pitch region is an important cue for back channeling production in Japanese. Accordingly, he suggested a well-made system, which produces a back channel item after a low pitch region of a certain frequency. Ward \& Al Bayyari (2007) have also introduced various prosodic features in the speakers' speech signaling the appropriate times of back channeling in Egyptian Arabic, including a pitch upturn at the phrasal end, low flat pitch associated with a lengthened vowel at dis-fluency points, and a sharp pitch downslope.

\section{Methods}

All of the research results mentioned above highlighted the importance of investigating back channel responses. In this paper, the focus is on the Persian back channels in formal and informal contexts. The feedback phenomena studied are words, phrases, and utterances, which serves the function of managing communicative interaction. The question we are seeking to answer is: "what is the effect of formality on the use of back channel responses?" to answer this question, we conducted a comparative analysis of Persian telephone conversations to examine frequency, type, function and placement of back channel responses in formal and informal communicative settings. 30-min phone conversations conducted in total in informal settings and 30-min phone conversations conducted in total in formal settings were examined. Each 30-min was consisted of two conversations lasting $15 \mathrm{~min}$. In formal contexts, the participants were employees in Tehran and Shiraz companies, discussing their contracts, problems and benefits. In informal contexts, one conversation was between a brother and a sister talking about an email, and the other conversation was between two close friends discussing one of their memories. The participants' age vary between 25 to 35.The study of telephone conversations in the examination of back channel responses is beneficial in many respects. As Heinz (2003) asserted, back channel responses are more salient in phone conversations, because participants in phone conversations lack access to non-verbal cues other than voiced related features, such as pitch. Ward and Tsukahara's definition (2000) of back channel feedback was used in this study. They define a back channel signal as a response directly to the content of the utterance of the other which is optional and does not require acknowledgment by the other. The data are extracted from the Farsdot data-base of Persian telephone conversations with the allowance of Guyeshpardaz institute. These phone conversations with various topics are collected and categorized for natural language processing. The contexts were distinguished as formal and informal based on the relationship between the participants, the form of language they used and the power relation. Back channels in this paper are considered as a response directly to the content of the utterance and do not require acknowledgment by the other. With this definition, the corpus contains 363 back channel responses, 233 in formal context, and 130 in informal context.

\section{Discussion and Results}

The overall frequency counted for back channels across the 30-min interaction of formal context was 233, but in informal context, back channel behavior occurred less frequently, 130 times. However, this difference was attributable to the different conversational topics. In formal setting, the participants talked about business and took long turns, so the opportunity of back channeling 
enhanced for the listener, but in informal setting the speaker took short turn and the listener had less time to produce back channel responses and more time to become a speaker.

In daily human communication, back channels involve the use of multi-modal expression, which means that it can be expressed by means of verbal and non-verbal expressions. As far as phone conversations were the focus of the investigation in this paper, and since the participants lacked access to non-verbal cues, only verbal items were examined. The only non-verbal back channel examined in this paper is laughter, because it has its own pitch which makes it distinguishable from the speech. We considered laugh as a back channel, since it appears in a back channel position and, based on the definition, it is a direct response to the content of the speaker's utterance and also it is optional and does not require acknowledgment. There appeared to be an agreement on the fact that verbal back channel responses could be expressed both by means of lexical and non-lexical utterances. Lexical utterances consisted of words such as /bale/ (yes), /doroste/(right), /âre/(yeah), /âfarin/(excellent), /ahsant/(perfect), /xob/(okay), /jedDan/ (really), short utterances like /duruqmigi/(kidding me), /sad dar sad/(100 percent), even longer expressions consisting of repetition of what the speaker had just said, anticipation or completion of the speakers contribution and short questions or request for clarification. Non-lexical back channels in the corpus of this study are sounds such as /âhâ/, /hmm/, /hâ/, /ee/, /vây/.

In both contexts, the ranking of the types of back channels with respect to frequency was partly the same. The overwhelming majority of back channels in both contexts were lexical back channels:

\begin{tabular}{|c|c|c|}
\hline Types of BC & Informal context & Formal context \\
\hline Lexical BC & 70 & 80 \\
\hline Non-lexical BC & 30 & 20 \\
\hline
\end{tabular}

Table 1: The frequency of different types of BC

Within the category of lexical back channels, there were significant differences between the use of four items: /bale/ (yes), /doroste/(right), /xob/(okey), /âre/(yeah). The distinction between these short tokens can be described as one of the politeness or formality. As demonstrated below in example 1 and 2, / bale/ and /doroste/are used in formal context with polite verbal form, while in example 3 and 4, /xob/and /âre/are used with informal and casual speech.

1) A: hatâ sabzijât ro va mivejâte tâze ro be moqe be unjâ even vegetables $\mathrm{OBJ}$ and fruits fresh $\mathrm{OBJ}$ in time to there

miresundan va dar unjâ tozi: mikardan transmit-3SG and in there distribute helping.verb-3SG

they even transmitted fresh vegetables and fruits there and distriuted them

B: doroste

Right

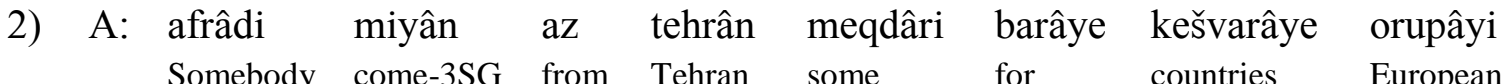




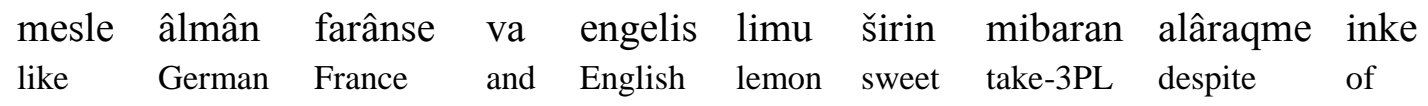

$\begin{array}{llll}\text { nemišnâsan } & \text { limu } & \text { širin } & \text { ro } \\ \text { not-know-3PL } & \text { lemon } & \text { sweet } & \text { OBJ }\end{array}$

Somebody came from Tehran and took some sweet lemon to European countries like German, France, and English; despite they do not know the sweet lemons

B: $\quad$ bale

In the examples 1 and 2, two agriculture engineers are talking about the condition of the fruit exportation, and the form /bale/and /doroste/are used to save the social face of the other person in conversation. Hence, the use of $/ x o b /$ or /âre/in the first two examples could be a threat to the social face of the speakers. In fact, the speaker's utterances are in polite style and are marked overtly with the use of complete form " va"(and), instead of its casual form " $o$ " (nd). In casual Persian conversations, /va/ (and) is usually contracted to the vowel /o/ and is attached to the last consonant of the previous word. In casual Persian conversations, /va/ "and" is usually contracted to the vowel $/ \mathrm{o} /$ and attached to the last consonant of the previous word. If the examples were more colloquial, then one would be less likely to find/bale/and /doroste/, but more likely to find /xob/and /âre/as a back channel response. Interestingly, the most frequent type of back channels used in formal phone conversations was /bale/and after that /doroste/.

3) A: ye dune az čârtâ ro mituni bezani xob

one out of four OBJ can-2SG choose-2SG okay

you can choose one of the four options, okay

B: $x o b$

Okay

$\begin{array}{lllll}\text { 4) A: fardâ } & \text { bâyad } \\ \text { Tomorrow } & \text { must } & \text { call } & \text { bezanam } & \text { bebinam } \\ \text { helping.verb.1SG } & \text { see.1SG }\end{array}$

rafteš mašad yâ na

went.3SG Mashhad or not

Tomorrow, I must call him/her to see whether s/he went to Mashhad or not

B: âre

Yeah

In the example 3, two friends are taking about the details of an email, the first $/ x o b /$, in the first turn, count as a short emphatic question to ensure that the listener is following, but the second 
/xob/function as a back channel which means that "I have understood thus far, please continue". âre/in example 4 also serves the same function.

In the following tables, the frequency of short utterances functioning as back channels is evident. It is convincible that some sort of colloquial use of linguistics items which function as back channel, are not used in formal setting. For instance, /duruqmigi/ (kidding me) acts as a back channel signaling surprise in conversation, but is not used in formal context.

\begin{tabular}{|c|c|}
\hline Bale (yes) & $52 \%$ \\
\hline Doroste (right) & $25 \%$ \\
\hline âhâ (non-lexical BC) & $7 \%$ \\
\hline Hmm (non-lexical BC) & $6 \%$ \\
\hline repetition & $4 \%$ \\
\hline Âfarin (excellent) & $2 \%$ \\
\hline âre (yeah) & $1 \%$ \\
\hline sad dar sad (100 percent) & $1 \%$ \\
\hline Suggestion & $0.5 \%$ \\
\hline Laughter & $0.5 \%$ \\
\hline Hâ (non-lexical BC) & $0.5 \%$ \\
\hline Ahsant (perfect) & $0.5 \%$ \\
\hline
\end{tabular}

Table 2: The frequency of different types of BCs in formal setting

\begin{tabular}{|c|c|}
\hline Xob (okay) & $46 \%$ \\
\hline Hâ (non-lexical BC) & $16 \%$ \\
\hline Hmm (non-lexical BC) & $12 \%$ \\
\hline ee (non-lexical BC) & $9 \%$ \\
\hline Âre (yeah) & $8 \%$ \\
\hline doruqmigi (kidding me) & $4 \%$ \\
\hline Laughter & $3 \%$ \\
\hline Vây (non-lexical BC) & $0.5 \%$ \\
\hline Repetition & $0.5 \%$ \\
\hline Jedan (really) & $0.5 \%$ \\
\hline Bale (yes) & $0.5 \%$ \\
\hline
\end{tabular}

Table 3: The frequency of different types of BCs in informal settings

In accordance with Wannaruk (1997) \& Maynard (1986)and based on our analysis, we categorized the functions of back channels in seven categories as follows: 
1. Agreement: the listener provided a back channel to show agreement with the speaker, when the speaker talks about ideas or known facts.

2. Understanding: it signaled the listeners' understanding, when the speaker says something unknown to the listener or when confirmation of the listeners' understanding is necessary.

3. Continuer: it functioned as an encouragement for the speaker to complete his/her turn especially when there is a problem on the part of the speaker for recalling the events.

4. Surprise: the listener indicated his surprised.

5. Emotional response: when the listener showed his sorrow, pity, happiness.

6. Misunderstanding: the listener misunderstood or mishear what he has heard and and showed his attitude by back channel production.

7. Disagreement: it showed the listeners' different opinion.

The frequency of these categories was examined in both contexts. In formal context, the most frequently displayed function of back channel was agreement, the higher frequency of this function is attributable to the high frequency of / bale/ and /doroste/ in formal context, which serves as an agreement and acceptance of the speakers' speech. Interestingly, back channels with this function are also used more in formal setting. In our corpus, for instance, short utterances like /ahsant/(perfect), /âfarin/(excellent), occur in formal context.

Conversely, in informal context, because of more frequent use of $/ \mathrm{xob} /$ and /âre/ which function as a claim of understanding, understanding appears to be the most frequent function of back channels. Furthermore, based on the findings, back channels with the pragmatic meaning of surprised and emotional responses are more frequent in informal rather than formal context. Table 4 and 5 showed the frequency of different function of back channels in both contexts.

\begin{tabular}{|c|c|}
\hline Functions of BCs & Frequency \\
\hline Agreement & $45 \%$ \\
\hline Understanding & $41 \%$ \\
\hline Continuer & $13 \%$ \\
\hline Surprise & $0.5 \%$ \\
\hline Emotional response & $0.5 \%$ \\
\hline
\end{tabular}

Table 4: The frequency of back channels functions in formal setting

\begin{tabular}{|c|c|}
\hline Functions of BCs & Frequency \\
\hline Understanding & $54 \%$ \\
\hline Agreement & $16 \%$ \\
\hline Misunderstanding & $12 \%$ \\
\hline Continuer & $12 \%$ \\
\hline Surprise & $12 \%$ \\
\hline Emotional response & $3 \%$ \\
\hline Disagreement & $0.5 \%$ \\
\hline
\end{tabular}

Table 5: The frequency of back channels functions in informal setting

Based on White (1986) \& Wannaruk (1997), Back channel responses mostly occur at the following locations: 
1. Clausal units: most of back channels occur at clausal units, partly, because at the end of syntactic units, the semantic content is almost complete, so the listener wants to show his/her understanding or perception o these bits of information with producing back channel signals.

2. Falling pitch region: as in English and Japanese, our analysis shows that a falling pitch region is a cue which shows the listener the time of back channeling.

3. Short emphatic questions: these short emphatic utterances, such as /xob/ (okay), /âre/ (yeah), /mage na/ (isn't it), come at the end of speakers' utterance and functions as a cue for the speaker to ensure that the listener is following the process of conversation.

Based on the analysis, in both contexts, back channels occurred frequently after clausal units and falling pitch region. However, during the prosodic analysis we understood that most of the time clausal units co-occurred with a falling intonation. Moreover, the findings showed that there were statistically significant differences in the use of short emphatic questions in the speakers' utterance in various contexts. These utterances occur mostly in informal context, where the relation between the participants is friendlier. As a result, back channels occurred after these utterances more in informal context.

$\begin{array}{lllllll}\text { 5) A: man } & \text { aslan un mozu: } & \text { ro } & \text { dust } & \text { nadaram } & \text { xob } \\ \text { I } & \text { at-all that topic } & \text { OBJ like helping.verb-1SG } & \text { okay }\end{array}$

I do not like that topic at all, okay

B: $x o b$

Okay

\section{Summary}

The present study has revealed some differences in the frequency, type, function, and placement of back channels used in different Persian conversational setting. These differences are attributable to the level of formality or politeness and have important implications for Persian learners. As we have demonstrated above, the back channels /bale/ and /doroste/ are not used with the informal speech style, inversely the usage of $/ x o b /$ and /âre/in formal conversation seem impolite. We have identified seven functions for Persian back channel responses and compared these functions in both contexts. The results showed that the agreement function was used more in formal context due to the higher frequency of /bale/and /doroste/. On the other hand, because of the frequent use of $/ x o b /$ and /âre/in informal settings, the understanding function was more frequent. Given these differences, back channel signaling agreements were used more frequently in formal settings. Furthermore, with respect to the possible places of back channels in conversations, short emphatic questions occurred mostly in informal context, so they count as a possible place of back channels only in informal context. 


\section{Abbreviations}

1: First Person; 2: Second Person; 3: Third Person; OBJ: Object Marker; PL: Plural; SG: Singular

\section{References}

Angles J. et al. 2000, Japanese responses hai, eeand un: yes, no, and beyond, Language of communication, 20: 55-86.

Coates, J. 2003, Women, men and languages, A sociolinguistics account of gender differences in languages. Harlow: Pearson education limited.

Dixon J.A. \& Foster D.H. 1998, Gender, social context, and backchannel responses, Journal of social psychology, 138:134-136.

Fries C.C. 1952, The structure of English. New York: Harcourt Brace.

Heinz B. 2003, Backchannel responses as strategic responses in bilingual speakers' conversations, Journal of pragmatics, 35: 1113-1142.

Kim S. 2009, Pause fillers and back channels in Japanese and Korean discourse, Proceeding (NFLRC), 47: 30-37.

Kok I. \& Heylen D. 2010, Differences in listener responses between procedural and narrative tasks, Proceedings of the $2^{\text {nd }}$ international workshop on social signal processing, Italy: 5-10.

Li H.Z. 2006, Back channel response as misleading feedback in intercultural discourse, Journal of intercultural communication research, 35: 99-116.

Li H.Z. et al. 2010, Back channel responses and enjoyment of the conversation: the more does not necessarily mean the better, International journal of psychological studies, $2: 25-35$.

Maynard, S. K. 1986, On back channel behavior in Japanese and English casual conversations, Linguistics, 24: 1079-1108.

Tao H.Y. \& Thompson S.A. 1991, English backchannels in Mandarin conversations: A casestudy of super stratum pragmatic interference, Journal of pragmatics, 16: 209-223.

Wannaruk A.1997, Back channel behavior in Thai and American casual telephone conversations, Suranaree journal of science and technology, 4: 168-174.

Ward N.1996, Using prosodic clues to decide when to produce back channel utterances. International Conference on spoken language processing, Journal of pragmatics: 1728-1731.

Ward N. \& Al Bayyari Y. 2007, A prosodic feature that invites back channels in Egyptian Arabic, Perspectives in Arabic Linguistics xx. Amsterdam: John Benjamins: 186-206.

Ward N. \& Tsukahara W. 2000, Prosodic features which cue back channel responses in English and Japanese, Journal of pragmatics, 32: 1177- 1207.

White R. 1997, Back channeling, repair, pausing, and private speech, Applied linguistics, 18: 314-349.

White S. 1986, Functions of back channels in English: A cross cultural analysis of Americans and Japanese. Unpublished doctoral dissertation, Georgetown University.

Yngve V. 1970, On getting a word in edgewise. Papers from the $6^{\text {th }}$ regional meeting of Chicago linguistics society. University of Chicago, Chicago: 568-578. 
Authors' Contact Information:

Shahla Sharifi

Associate Professor, Linguistics department, Ferdowsi University of Mashhad, Iran shahlasharifi@hotmail.com

Mahnaz Azadmanesh

MA student of linguistics, Ferdowsi University of Mashhad, Iran

Mahnaz.azadmanesh@gmail.com 